\title{
ASTROINFORMATICS
}

DOI: http://dx.doi.org/10.18524/1810-4215.2017.30.114411

\section{ON THE CONCEPT OF THE ENHANCED FON CATALOG COMPILATION}

\author{
V. Andruk ${ }^{1}$, Q. Yuldoshev ${ }^{2}$, I. Eglitis ${ }^{3}$, L. Pakuliak ${ }^{1}$, A. Mullo-Abdolov ${ }^{4}$, \\ I. Vavilova ${ }^{1}$, Yu. Protsyuk ${ }^{5}$, H. Relke ${ }^{6}$, V. Golovnia ${ }^{1}$, S. Shatokhina ${ }^{1}$, O. Yizhakevych ${ }^{1}$, \\ Sh. Ehgamberdiev ${ }^{2}$, M. Muminov ${ }^{7}$, G. Kokhirova ${ }^{4}$, L. Kazantseva ${ }^{8}$ \\ ${ }^{1}$ Main Astronomical Observatory NASU,Kyiv, Ukraine, andruk@mao.kiev.ua \\ ${ }^{2}$ Ulugh Beg Astronomical Institute UAS,Tashkent, Uzbekistan, q.astrin@gmail.com \\ ${ }^{3}$ Institute of Astronomy, University of Latvia,Riga, Latvia, ilgmars@latnet.lv \\ ${ }^{4}$ Institute of Astrophysics of AS of Republic Tajikistan, Dushanbe, Tajikistan, \\ aziz.sherzod@gmail.com \\ ${ }^{5}$ Research Institute Mykolaiv Astronomical Observatory, Mykolaiv, Ukraine, \\ yuri@mao.nikolaev.ua \\ 6 Walter Hohmann Observatory,Essen, Germany, helena_relke@yahoo.com \\ ${ }^{7}$ Andijan State University,Andijan, Uzbekistan, muminov1951@gmail.com \\ 8 Astronomical Observatory of Kyiv Shevchenko National University, Kyiv, Ukraine, \\ kazl@ukr.net
}

\begin{abstract}
We suppose to compile the enhanced version of FON (Photographic Survey of the Northern Sky) catalog of stellar positions and B-magnitudes in the sky region from -20 to 90 degrees on declination. The photographic base of the project comprises about 5,700 plates obtained at three observatories of the former SU MAO NAS of Ukraine, Kitab observatory of Uzbekistan and Gissar observatory of Tajikistan. The mean epoch of the catalog is around 1988. The expected limited stellar magnitude is $17.5^{m}$. More than 5,400 plates are processed to support the photometric part of the catalog. The plates were shot on the $1.2 \mathrm{~m}$ Schmidt telescope in Baldone, Latvia, in the $\mathrm{U}$ and $\mathrm{V}$ bands. Color data provide the possibility of catalog correction for the photometric color equation. Stars brighter than $\mathrm{V}<8.5^{m}$ will be supplemented with photoelectric U, B, V data. Proper motions will be obtained using GAIA data.
\end{abstract}

Keywords: star catalogs, digital image processing, photometry, UBV photometric system.

\section{Introduction}

In 1976 two scientists of MAO NAS of Ukraine I.G.Kolchinsky and A.B.Onegina proposed the project of the photographic survey of the northern sky (FON) (Kolchinsky \& Onegina,1977). The idea arose after the acquisition of similar wide-angle astrographs of Carl Zeiss company with focal length 2 or 3 meters and aperture $40 \mathrm{~cm}$ in several observatories of the former USSR. The observational part of the project should have been run at 6 observatories. They are Kiev (Ukraine), Kitab (Uzbekistan), Zelenchuk (Russia), Abastumani (Georgia), Zvenigorod (Russia), Dushanbe (Tajikistan). Regular observations of the northern sky started in 1982. Unfortunately, for objective reasons, the idea of the four-fold overlapping of the sky with 6 astrographs was not fully implemented (Pakuliak et al., 2016). Here, in the final version of the enhanced catalog, we suppose to use plates from collections of three observatories from the previous list, namely Kiev, Kitab, and Dushanbe. In 2016 the processing of 5,400 plates was started for the providing the photometric part of the catalog with $\mathrm{U}, \mathrm{V}$ magnitudes (Eglitis \& Eglite, 2017b). Plates were shot on the 1.2 m. Schmidt telescope in Baldone, Latvia.

In MAO NAS of Ukraine, the FON project is carried out in the framework of UkrVO project (Vavilova et al., 2012a; 2012b; 2017; Vavilova, 2016).

\section{Current status of the project}

By now we have created the catalog of positions and B-magnitudes of stars for the Northern hemisphere (declination from $-4^{\circ}$ to $90^{\circ}$ ). The observational base of the catalog comprises 2260 digitized plates of MAO NASU 2-meters Carl Zeiss astrograph (Akhmetov, 2016; Andruk et al., 2014; 2015a; 2015b; 2016a; 2016c; Protsyuk, 2015a; Protsyuk \& Relke, 2016). 
Plates were digitized using two commercial scanners, Microtek ScanMaker 9800XL TMA and Epson Expression 10000XL. The resolution of images is $1200 \mathrm{dpi}$, linear dimensions of the most plates are $30 \times 30 \mathrm{sm}$ or 13,000x13,000 pixels (Protsyuk et al., 2014a; 2014b; 2014c). The catalog contains 24.7 million of stars and galaxies brighter than $\mathrm{B}=16.5^{\mathrm{m}}$. The mean epoch of the catalog is 1988.2. Positions of catalog entries are given in the TYCHO-2 reference system, B-magnitudes are obtained in the system of photoelectric standards. The internal accuracy of the catalog for all objects is $\sigma_{\alpha \delta}= \pm 0.28 "$ and $\sigma_{B}= \pm 0.17^{m}$ (for stars in the interval $\mathrm{B}=7^{m}-14^{m}$ these errors are $\sigma_{\alpha \delta}= \pm 0.13$ " and $\sigma_{B}$ $\left.= \pm 0.08^{m}\right)$. The convergence between calculated and reference positions is $\sigma_{\alpha \delta}= \pm 0.06 "$ and convergence with photoelectric B-magnitudes is $\sigma_{B}= \pm 0.14^{m}$ (Andruk et al., 2016b).

The result of the processing the Kitab FON photographic collection containing 1963 plates in the declination band from -20.5 to +2.5 degrees is the catalog of positions and B-magnitudes of 13.4 million stars and galaxies with $\mathrm{B} \leq 17.5^{\mathrm{m}}$ for the mean epoch 1985.0. (Yuldoshev et al., 2016a; 2016b). The digital images of plates were obtained on Epson Expression 10000XL commercial scanner with the same parameters of scanning (Muminov et al., 2013; 2014; 2017). Catalog positions are in the TYCHO-2 reference system and Bmagnitude in the system of photoelectric standards. The internal accuracy of the catalog for all objects is $\sigma_{\alpha \delta}= \pm 0.23$ " and $\sigma_{B}= \pm 0.15^{m}$ (for stars with B in the interval from $7^{m}$ to $14^{m}$ the errors are $\sigma_{\alpha \delta}= \pm 0.085$ " and $\left.\sigma_{B}= \pm 0.054^{m}\right)$. The convergence between calculated and reference positions is $\sigma_{\alpha \delta}= \pm 0.042$ " and convergence with photoelectric B-magnitudes is $\sigma_{B}=$ $\pm 0.16^{m}$ (Yuldoshev et al., 2017).

Kiev and Kitab FON components have the overlapping area on declination from -4 to +2.5 degrees $(\mathrm{Mu}-$ minov et al., 2016). After the cross-identification of objects in two catalogs, the compiled catalog was created. The compiled catalog contains 36.7 million of stars and galaxies referred to the mean epoch 1987.0. For the objects measured twice or more times the errors are $\sigma_{\alpha \delta}= \pm 0.26 "$ and $\sigma_{B}= \pm 0.17^{m}\left(\sigma_{\alpha \delta}= \pm 0.120 "\right.$ and $\sigma_{B}= \pm 0.072^{m}$ for stars in the interval $\mathrm{B}=7^{m}$ $\left.-14^{m}\right)$.

Dushanbe (Gissar) FON glass collection of the Institute of Astrophysics, the Academy of Sciences of Republic Tajikistan comprises near 1560 plates (MulloAbdolov et al., 2017). Before the start of plate collection digitizing, the commercial scanner Microtek ScanMaker 1000XL Plus has been investigated. The estimated errors of the scanner for stars brighter than $\mathrm{B} \leq 13.5^{m}$ are $\sigma_{x} y= \pm 0.054 \mathrm{px}$ and $\sigma_{m}= \pm 0.020^{m}$ for rectangular coordinates and instrumental magnitudes respectively. The estimated astrometric accuracy obtained from the processing of the test plate with Pleiades in the system of TYCHO-2 is $\sigma_{\alpha \delta}= \pm 0.13$ ".
To enhance the photometric part of the catalog the collection of photographic plates shot on the $1.2 \mathrm{~m}$. Schmidt telescope in Baldone, Latvia, in the $U$ and $\mathrm{V}$ color bands are actively digitized and processed (Eglitis et al., 2016b; 2016c). The collection comprises near $780 \mathrm{U}$ and 4660 Vplates. To date, the rectangular coordinates and photometric data of registered objects are obtained for almost the third part of the collection. The plate digitizing is made on the Epson Expression 10000XL scanner (Eglitis \& Andruk, 2017a). It was tested for positional and photometric errors of the developed method of image processing by comparison between images with 8 and 16-bit grey scale color range. The instrumental errors found from the testing are $\pm 0.0026 \mathrm{px}$ and $\pm 0.0024^{m}$ for coordinates and magnitudes respectively. To estimate the astrometric and photometric accuracy, we processed 6 consecutive scans of the same plate with 1200 dpi resolution. The errors obtained for stars brighter than $13.5^{\mathrm{m}}$ in U-band are $\sigma_{x} y= \pm 0.021 \div 0.027 \mathrm{px}$ and $\sigma_{m}=$ $\pm 0.014^{m} \div 0.016^{m}$.

\section{The photometric system of the catalog}

The observational material in FON observational program was shot on refractors. The photometric data obtained as a result of digital image processing are distorted with the color equation, i.e.calculated photographic $B_{p h}$ magnitudes are burdened with systematic errors depending on B-V color indices of stars. To obtain $B_{p h}$ magnitudes, the characteristic curves were used, calibrated by reference stars with photoelectric data $B_{p e}$ (Kornilov et al., 1991; Mermilliod, 1991; Andruk et al., 1995; 2017; Relke et al., 2015). The results of the comparison of photometric differences $\Delta \mathrm{B}=B_{p h^{-}}$ $B_{p e}$ for calculated and reference stars are shown in Fig.1.

Panels $\mathbf{a}$ and $\mathbf{c}$ show the trend of differences $\Delta \mathrm{B}$ with a stellar magnitude $B_{p e}$. Panels $\mathbf{b}$ and $\mathbf{d}$ demonstrate the dependence of differences $\Delta \mathrm{B}$ on photoelectric values $\mathrm{B}-\mathrm{V}$ for Kiev and Kitab components of FON catalog. The number of compared stars in catalogs is 29,776 and 6,135 for corresponding plots. RMS errors $\mathrm{m}$ of magnitude differences are $0.140^{\mathrm{m}}$ and $0.156^{m}$ respectively. The presence of a color equation is noticeable on the panels $\mathbf{c}$ and $\mathbf{d}$. Its value is approximately $0.16(\mathrm{~B}-\mathrm{V})$ of stellar magnitude.

\section{Summary}

In nearest perspective we plan the next steps:

1. Until 2019 to digitize and process the plates from the Dushanbe FON collection. To create the catalog of positions and B-magnitudes. 

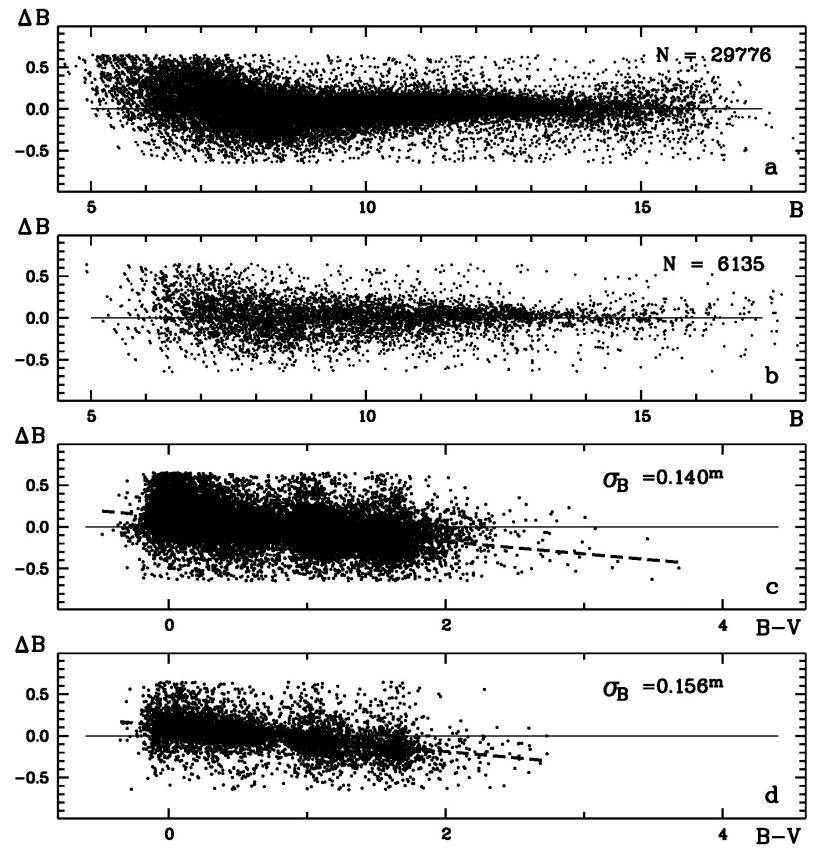

Figure 1: Photometric systems of FON catalog components

2. Until 2020 to complete the digitizing and processing of $\mathrm{U}$ and $\mathrm{V}$ plates from the collection of 1.2 Schmidt telescope in Baldone. To obtain catalogs of positions and $\mathrm{U}, \mathrm{V}$ magnitudes.

3. To supply the compiled catalog with bright stars data (down to $\mathrm{V}=0^{m}$ ) and to make the correct replacement for stars with $\mathrm{V}<8.5^{m}$ with photoelectric U, B, V data.

4. To correct B-magnitudes of the compiled catalog for the color equation.

5. Using GAIA data to obtain proper motions of stars.

Fig. 2 demonstrates the distribution of stars from the catalog with $=10 \pm 0.5^{m}$ on the hemisphere with the visible concentration of stars in the areas of Milky Way and the regions with the absorption of light in them.

The software created in the process of compilation Kiev and Kitab parts of the catalog was testes also in the processing of plates with Solar System bodies to determine their positions.(Eglitis et al., 2016a; Kazantseva et al., 2015; Protsyuk et al., 2015b; 2017; Shatokhina et al., 2016; Vavilova et al., 2014; Yizhakevych et al., 2014; 2015; 2016; 2017).

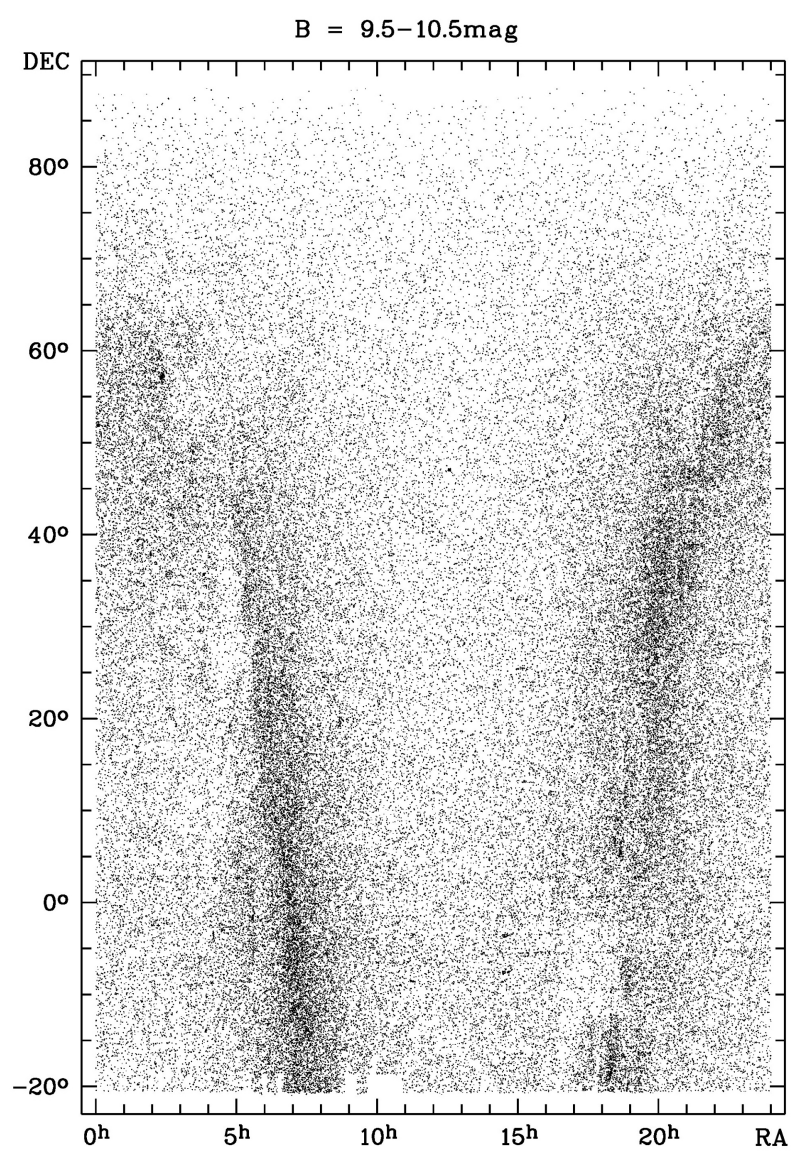

Figure 2: The star map on the data of enhanced FON catalog

\section{References}

Akhmetov V.S.: 2016, Odessa Astron. Publ., 29, 116. Andruk V., Kharchenko N., Schilbach E. et al.: 1995, $A N$, 316, 225.

Andruk V.N., Golovnya V.V., Ivanov G.A. et al.: 2014, Odessa Astron. Publ., 27, 53.

Andruk V.N., Pakuliak L.K., Golovnya V.V. et al.: 2015, preprint (arXiv:1512.05535).

Andruk V.M., Pakuliak L.K., Golovnia V.V. et al.: 2015, Odessa Astron. Publ., 28, 192.

Andruk V.M., Golovnia V.V., Ivanov G.A. et al.: 2016, Kinem. Phys. Cel. Bodies, 32, N1, 38.

Andruk V.M., Pakuliak L.K., Golovnia V.V. et al.: 2016, Kinem. Phys. Cel. Bodies, 32, N5, 260.

Andruk V., Pakuliak L., Golovnia V. et al.: 2016, Bull. T.Shevchenko Nat. Univ. Kyiv. Astron., N54, 24 (in Ukrainian).

Andruk V.M., Pakuliak L.K., Golovnia V.V. et al.: 2017, Science and Innovation, 13a, N1, 17.

Eglitis I., Eglite M., Shatokhina S.V. et al.: 2016, Odessa Astron. Publ., 29, 123. 
Eglitis I., Eglite M., Pakuliak L.K. et al.: 2016, Odessa Astron. Publ., 29, 126.

Eglitis I., Eglite M., Andruk V. et al.: 2016, Bull. T.Shevchenko Nat. Univ. Kyiv. Astron., N54, 21 (in Ukrainian).

Eglitis I., Andruk V.: 2017, Open Astronomy, 26, N1, 7 .

Eglitis I., Eglite M.: 2017, Science and Innovation, 13a, N1, 55.

Kazantseva L.V., Shatokhina S.V., Protsyuk Yu.I. et al.: 2015, Kinem. Phys. Cel. Bodies, 31, N1, 37.

Kolchinsky I.G., Onegina A.B.: 1977, Astrometry and Astrophysics, N33, 11 (in Russian).

Kornilov V.G., Volkov I.M., Zakharov A.I. et al.: 1991, Trudy GAIS, 63, 1 (in Russian).

Mermilliod J.C.: 1991, [online] Available at: http://vizier.cfa.harvard.edu/viz-bin/VizieR?source $=I I / 168$.

Mullo-Abdolov A., Kokhirova G., Relke H. et al.: 2017, Odessa Astron. Publ., 30, this issue.

Muminov M.M., Kahharov B.B., Yuldoshev K.H. et al.: 2013, Izvestija GAO. Pulkovo, 220, 517.

Muminov M.M., Yuldoshe Q.X., Ehgamberdiev Sh.A. et al.: 2014, Odessa Astron. Publ., 27, 57.

Muminov M.M., Ehgamberdiev Sh.A., Latipov A.A. et al.: 2016, Izvestija GAO. Pulkovo, 223, 339.

Muminov M., Yuldoshev Q., Ehgamberdiev Sh. et al.: 2017, Bulgarian Astron. J., 26, 1.

Pakuliak L.K., Andruk V.M., Golovnia V.V. et al.: 2016, Odessa Astron. Publ., 29, 132.

Protsyuk Yu.I., Andruk V.N., Kazantseva L.V.: 2014, Odessa Astron. Publ., 27, 59.

Protsyuk Yu.I., Andruk V.N., Muminov M.M. et al.: 2014, Odessa Astron. Publ., 27, 61.

Protsyuk Yu.I., Kovylianska O.E., Protsyuk S.V. et al.: 2014, Odessa Astron. Publ., 27, 63.

Protsyuk Yu., Andruk V., Mazhaev A. et al.: 2015, Odessa Astron. Publ., 28, 202.
Protsyuk Yu., Yizhakevych O., Kovylianska O. et al.: 2015, Odessa Astron. Publ., 28, 204.

Protsyuk Yu., Relke E.: 2016, Odessa Astron. Publ., 29, 144.

Protsyuk Yu.I., Kovylianska O.E., Protsyuk S.V. et al.: 2017, Science and Innovation, 13a, N1, 58.

Relke E., Protsyuk Yu.I., Andruk V.M.: 2015, Odessa Astron. Publ., 28, 211.

Shatokhina S., Kazantseva L., Kazantsev A. et al: 2016, Odessa Astron. Publ., 29, 151.

Vavilova I.B., Pakulyak L.K., Shlyapnikov A.A. et al.: 2012, Kinem. Phys. Cel. Bodies, 28, N2, 85.

Vavilova I.B., Pakuliak L.K., Protsyuk Yu.I. et al.: 2012, Baltic Ast., 21, N3, 356.

Vavilova I., Golovnya V., Andruk V. et al.: 2014, Odessa Astron. Publ., 27, 65.

Vavilova I.B.: 2016, Odessa Astron. Publ., 29, 109.

Vavilova I.B., Yatskiv Ya.S., Pakuliak L.K. et al.: 2017, IAUS, 325, 361.

Yizhakevych O., Andruk V., Pakuliak L. et al.: 2014, Odessa Astron. Publ., 27, 67.

Yizhakevych O.M., Andruk V.M., Pakuliak L.K.: 2015, Odessa Astron. Publ., 28, 213.

Yizhakevych O.M., Andruk V.M., Pakuliak L.K.: 2016, Odessa Astron. Publ., 29, 155.

Yizhakevych O.M., Andruk V.M., Pakuliak L.K.: 2017, Kinem. Phys. Cel. Bodies, 33, N3, 142.

Yizhakevych O.,Andruk V., Pakuliak L. et al.: 2017, Bull. T.Shevchenko Nat. Univ. Kyiv. Astron., N55, 9 (in Ukrainian).

Yuldoshev Q.X., Muminov M.M., Ehgamberdiev Sh.A. et al.: 2016, Odessa Astron. Publ., 29, 160.

Yuldoshev K., Usmanov O., Egamberdiev E. et al.: 2016, Bull. T.Shevchenko Nat. Univ. Kyiv. Astron., N54, 28 (in Ukrainian).

Yuldoshev Q.X., Ehgamberdiev Sh.A., Muminov M.M. et al.: 2017, Kinem. Phys. Cel. Bodies, 33, N5, 250 . 